\title{
COMMEMORATING THE 80TH ANNIVERSARY \\ OF THE FOUNDING OF PSYCHOMETRIKA: INTRODUCTION BY THE GUEST EDITORS
}

\author{
WILLEM HEISER
}

LEIDEN UNIVERSITY

LAWRENCE HUBERT

UNIVERSITY OF ILLINOIS

The Psychometric Society was founded in 1935, with the first volume of the Society's journal, Psychometrika, appearing in 1936. This special section starts with a brief history of the formation of the Society and its journal in the early thirties, in which we give our version of a "creation narrative", while paying special attention to the important role of Paul Horst. Upon invitation of the President of the Society, Terry Ackerman, to commemorate Psychometrika's 80th anniversary, we decided to highlight the ten most frequently cited articles appearing in the journal from 1936 to the present (with an end date arbitrarily fixed on April 1, 2016). Commentaries were solicited from Psychometric Society members with expertise in the topics covered by each of the papers. These commentaries were presented during the Annual Meeting of the Society held in Asheville, North Carolina, on July 12 through July the 15th, 2016. The written versions of these presentations form the main part of the special section.

The commentaries are grouped into three themes, with a chronological ordering within these groups. The first and clearly major theme is "Solutions for Problems in the Factor Analysis Model". Half of the papers belongs to this group; it covers the rotation problem, the selection and testing of the number of factors, and measuring the simplicity of a factor structure. The second theme is "Analysis of Proximities", comprising two papers on multidimensional scaling (MDS) and one on hierarchical clustering. One of the MDS papers (by Kruskal) consisted in fact of two separate but linked parts published in two consecutive issues of Psychometrika, and the two parts are often cited together. That is why we considered them as one highly cited paper. The third and final theme is "Properties of Covariance Matrices in Multivariate Analysis". It contains the top-cited article, the one on Cronbach's alpha, which addresses the equivalence of different sets of items intended to measure the same attribute, and a paper testing the shapes of group profiles in repeated measures analysis of variance.

The three themes are easily recognized as three classic streams of research and development in psychometrics. Surprisingly perhaps, there are at least two other major streams of research in our field that did not appear among the ten most frequently cited articles: item response theory (IRT) and structural equation modeling (SEM). Several of our commentators note that one reason for a high citation frequency appears to be the amount of visibility and recognition acquired outside of the psychometric community, and another reason mentioned more than once is that some long-standing puzzle or controversy is resolved. Many other reasons for the absence of IRT and SEM easily come to mind. Therefore, in no way do we wish to create the suggestion that

Correspondence should be made to Willem Heiser, Leiden University, Leiden, The Netherlands. Email: heiser@fsw.leidenuniv.nl 
our selection of articles covers all important breakthroughs in the field. It is our hope that the reader finds inspiration in these ten prominent steps in the evolution of psychometric thinking, as brought alive by our expert commentators.

Manuscript Received: 27 OCT 2016

Published Online Date: 21 NOV 2016 